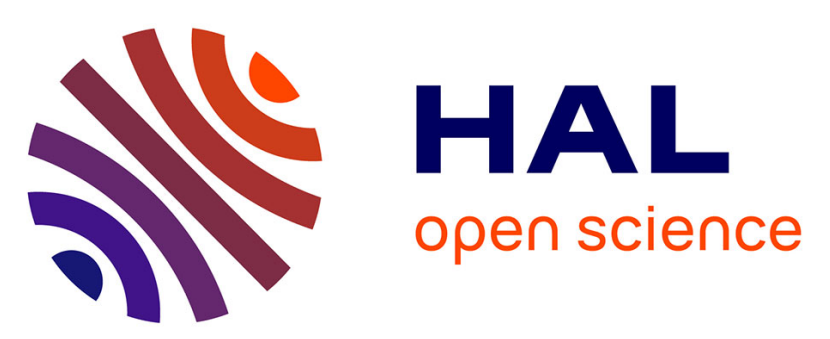

\title{
FRET detection in the plasma membrane using Total Internal Reflection Fluorescence Lifetime Imaging Microscopy
}

Pierre Blandin, Sandrine Leveque-Fort, Sandrine Lecart, Frédéric Druon, Patrick Georges, J.C Cossec, M.C. Pottier, Zsolt Lenkei

\section{To cite this version:}

Pierre Blandin, Sandrine Leveque-Fort, Sandrine Lecart, Frédéric Druon, Patrick Georges, et al.. FRET detection in the plasma membrane using Total Internal Reflection Fluorescence Lifetime Imaging Microscopy. CLEO (Conference on Lasers and Electro-Optics), May 2008, San José, United States. hal-00808701

\section{HAL Id: hal-00808701 https://hal.science/hal-00808701}

Submitted on 8 Apr 2013

HAL is a multi-disciplinary open access archive for the deposit and dissemination of scientific research documents, whether they are published or not. The documents may come from teaching and research institutions in France or abroad, or from public or private research centers.
L'archive ouverte pluridisciplinaire HAL, est destinée au dépôt et à la diffusion de documents scientifiques de niveau recherche, publiés ou non, émanant des établissements d'enseignement et de recherche français ou étrangers, des laboratoires publics ou privés. 


\title{
FRET detection in the plasma membrane using Total Internal Reflection Fluorescence Lifetime Imaging Microscopy
}

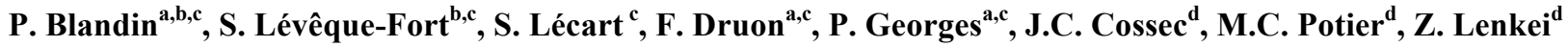 \\ ${ }^{a}$ Laboratoire Charles Fabry de l'Institut d'Optique, CNRS, Univ Paris-Sud, Campus de Polytechnique, 91127 Palaiseau cedex,France \\ ${ }^{b}$ Laboratoire de Photophysique Moléculaire, CNRS UPR3361, Univ Paris-Sud, 91405 Orsay France \\ ${ }^{c}$ Centre de Photonique Biomédicale, Univ Paris Sud, 91405 Orsay \\ ${ }^{d}$ ESPCI-CNRS UMR 7637, 75005, Paris
}

\begin{abstract}
We developed a Total Internal Reflection Fluorescence Lifetime Imaging Microscope to perform functional imaging of living cells membranes labeled with FRET couples. Förster Resonance Energy Transfer efficiency can thus be followed with subwavelength axial resolution.
\end{abstract}

Förster Resonance Energy Transfer has attracted a lot of attention in the past few years. Indeed, such progress has been made in both the methods and engineering of fluorescent probes that fluorescent protein-based FRET has become a powerful method to investigate the biology of the cell [1]. It is now possible to use FRET imaging to monitor protein-protein interactions, biochemical reactions, and polymer organization within living cells. The quantification of FRET is basically based on three methods: intensity, spectral and lifetime imaging [2]. We chose fluorescence lifetime imaging, because it is independent on concentration and intensity, and consequently it suffers less from photobleaching. By measuring the fluorescence lifetime of couple donor, we can estimate the efficiency of the transfer, and relate it to the distance between the two fluorophores and the subsequent biophysical phenomenon.

In some biomedical applications, imaging with high spatial resolution is required. For example, in neurobiology, localization of some membrane receptor gives crucial information about their trafficking and the neuronal structure. Total Internal Reflection Fluorescence Microscopy (TIRFM) is a very efficient and precise optical tool to observe the events on or just below the plasma membrane [3]. With TIRFM and by transfecting cell with FRET probes, one can also make functional imaging just in the membranes.

For all these reasons, we combine these attractive techniques and so developed a Total Internal Reflection Fluorescence Lifetime Imaging Microscopy (TIRFLIM) setup, which is applied to dynamic followings of membranous activity.

TIRFM combines the advantages of wide-field imaging, with a uniform excitation on a large area, reduced acquisition time (without scanning process), and subwavelength axial resolution. In the past few years, many commercial objectives with high numerical aperture (N.A.) have appeared, and have allowed to easily perform TIRFM with simpler setups in the so-called "through the objective configuration". To have a versatile setup compatible with a large range of fluorophores, we used a $15 \mathrm{MHz}$ picosecond supercontinuum fibre laser (Fianium SC450) producing a wide excitation spectrum in the visible band. To avoid infrared reinjection in the laser, two 400-900nm dielectric mirrors are used to filter the infrared part of the supercontinuum (fig 1). The beam diameter is enlarged thanks to a telescope to reach a diameter around $1 \mathrm{~cm}$.

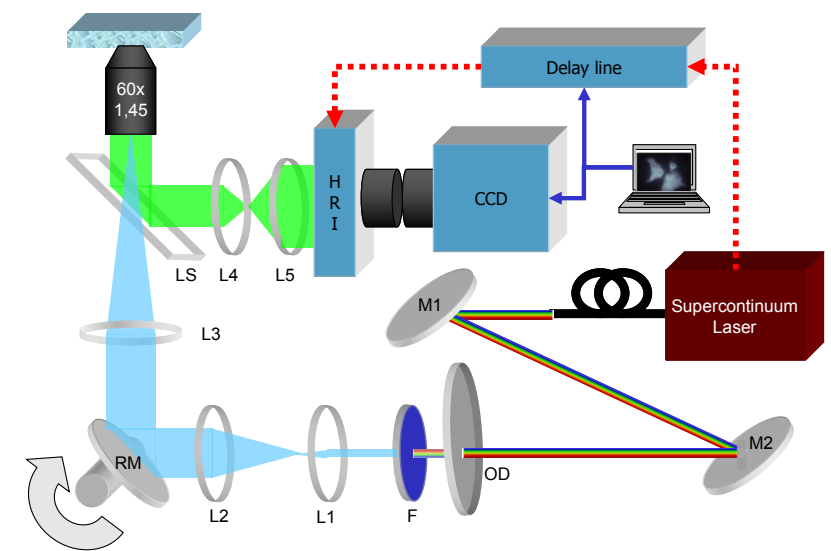

Figure 1 : experimental setup. M1, M2: plane broadband dielectric mirrors; OD: adjustable optical density; F: filter; L1:f=35mm; L2 and L3: $\mathrm{f}=$ 300mm; RM: rotating plane mirror; LS: dichroic beam splitter; L4: $\mathrm{f}=50 \mathrm{~mm}$; L5: $\mathrm{f}=150 \mathrm{~mm}$; Camera objectives: $\mathrm{f}=50 \mathrm{~mm}$.

An adjustable optical density (from 0 to 4 ) is placed on the beam to control the incident excitation power on the sample. The spectral range for the excitation is optimally adjusted using a filter set. A lens permits to focus the excitation beam in the back focal plane of the objective. A rotating plane mirror is positioned just before this lens to 
adjust the angle of the beam incident on the lens. The distance between the focus spot and the optical axis of the objective can be then adjusted to switch from classical wide field illumination to TIRF configuration and to control the penetration depth of the evanescent wave. To perform TIRFM, an objective with 1.45 N.A. (Olympus TIRFM, 60x) is used. Since TIRFM is a wide field microscopy technique, the fluorescence signal can be detected for field of typically $104 \mu \mathrm{m}^{2}$ with Charge Couple Device (CCD) cameras. And so the image of the sample is directly accessible just in single shot without scanning or computer processing, with diffraction limited transverse resolution. To preserve this liveliness, it seems relevant to measure fluorescence lifetime as well in wide field [4]. This FLIM measurement is achieved in time domain thanks to a High Rate Imager (HRI). The images from the HRI are optically relayed to a 12-bit CCD camera (Hamamatsu Orca AG) thanks to two camera objectives, with a magnitude of one (figure 1). With this FLIM technique, one can choose the optimal algorithm to measure the lifetime. The number of gates can be reduced (until only two gates in the case of Rapid Lifetime Determination algorithm or RLD [5]) according to the lifetime expected and the signal level. This procedure considerably decreases the acquisition time which is a crucial parameter in the investigation of dynamic processes. This HRI (Kentech Instrument Ltd) permits to open gates from 200 ps to $1 \mathrm{~ns}$ with increment of $100 \mathrm{ps}$ or larger gates of 4.8 or $10 \mathrm{~ns}$, essentially used for RLD acquisition. It is triggered to the laser via a delay line with increment down to $25 \mathrm{ps}$. Afterwards, by processing the time-gated intensity images pixel by pixel, the fluorescence decay is calculated and display in false colors.

Our setup is dedicated to two biomedical applications. The first biological process under investigation is the activation of cannabinoid receptors (CB1Rs) on the plasma-membrane, leading to down-regulation of cyclic Adenosine MonoPhosphate (cAMP) production in neurons. Our goal is to perform FRET-based detection of the mobilization of the cyclic AMP, by using a new FRET-based probe named AKAR. To validate the instrumental development and before beginning investigations on delicate primary neuronal cultures, TIRFLIM measurements have been performed in HEK293 cells, a standard and widely used cell line. HEK-293 cells are transfected with the AKAR-CFP/YFP probe (fig 2).
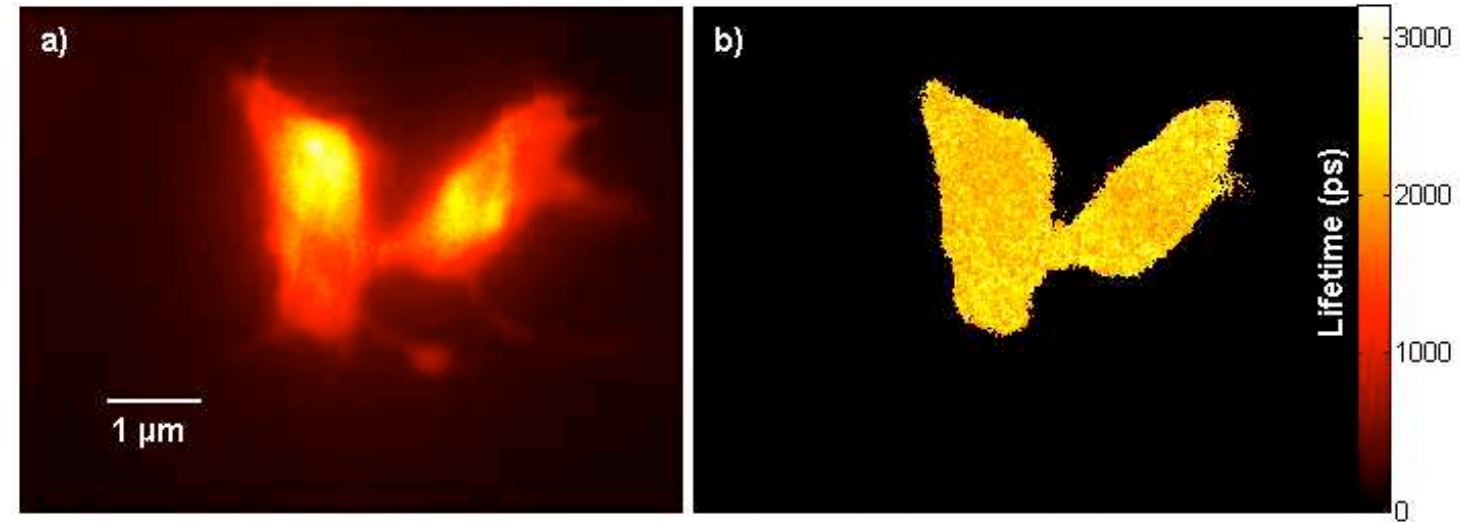

Figure 2 : (a) TIRF Intensity image and (b) TIRFLIM map of a HEK-293 cell transfected with the AKAR-CFP/YFP.

The second biological process studied is the homodimerisation of Amyloid Precursor Protein (APP), a membrane protein involved in Alzheimer's disease. We use APP labelled with CFP and with YFP. By transfecting HEK 293 cells with both, we can observe the homodimerisation of APP thanks to the subsequent FRET between CFP and YFP.

In conclusion, we demonstrate the instrumental development of a TIRFLIM setup which allows performing wide-field fluorescence lifetime imaging with a sub-wavelength axial resolution for a large range of fluorophores within few seconds of acquisition. We will also present the first results obtained with FRET-based detection in the biological processes under investigation.

Acknowledgements: This work was supported by the program Pôle Laser from the Contrat Plan Etat Région (20002006) and the MRCT from the CNRS.

\section{References}

[1] R. B. Sekar et al., "Fluorescence resonance energy transfer microscopy imaging of live cell protein localizations", JCB 160, 629 (2003)

[2] S. Pelet et al., "Comparing the quantification of Förster resonance energy transfer measurement accuracies based on intensity, spectral, and

lifetime imaging", Journal of Biomedical Optics 11, 034017 (2006).

[3] D. Axelrod, "Total Internal Reflection Fluorescence Microscopy in Cell Biology", Traffic 2, 764-774 (2001).

[4] S. Lévêque-Fort et al., "Fluorescence lifetime imaging with a low repetition rate passively mode-locked diode-pumped Nd:YVO4 oscillator", OL 30, $168-170$ (2005).

[5] R. Cubeddu et al., "Time-resolved fluorescence imaging in biology and medicine”, J. Phys. D: Appl. Phys. 35, R61-R76 (2002). 